Obviously, $\Lambda_{1}=\omega^{2}$, where $\omega$ is defined by (3.1).

A solution of the foregoing equation satisfying the initial conditions (4.2) is the cosine-type Jacobian elliptic function

$$
\tau(t)=c n\left(\omega^{*} t, k^{*}\right)
$$

with

$$
\begin{aligned}
\omega^{*} & =\left[\Lambda_{1}+\Lambda_{3}(A / h)^{2}\right]^{1 / 2} \\
k^{* 2} & =\frac{\Lambda_{3}(A / h)^{2}}{2\left[\Lambda_{1}+\Lambda_{3}(A / h)^{2}\right]}
\end{aligned}
$$

Since the period of the linear vibrations is $T=2 \pi / \Lambda_{1}^{1 / 2}$ the ratio of periods of nonlinear and linear vibrations is obtained as

$$
\frac{T^{*}}{T}=\frac{2 K}{\pi\left[1+\left(\Lambda_{\mathrm{z}} / \Lambda_{1}\right)\right.} \overline{\left.(A / h)^{2}\right]^{1 / 2}}
$$

In Fig. 3, the relation (4.6) is plotted for two types of orthotropy discussed previously and, for the sake of comparison, also for the isotropic case. For definiteness, it is assumed $\alpha=h / a=0.01$, and posed $j=10$ and $\lambda=40$. An inspection reveals a known sharp decrease of the period of vibrations with an increasing amplitude. Also, graphs for isotropic case and for the II type of orthotropy are plotted assuming $j=4$ and $\lambda=2$. Apparently, the mode patterns influence the period of the nonlinear vibrations more than the degree of anisotropy (at least for the two types of orthotropy considered).

\section{References}

1 Nowinski, J., "Response of a cylindrical shell to transverse nonlinear oscillations," TR 9, Dept. Mech. Eng., Univ. Delaware (May 1962)

${ }^{2} \mathrm{Chu}, \mathrm{H} .-\mathrm{N}$. "Influence of large amplitude on flexural vibrations of a thin circular cylindrical shell," J. Aerospace Sci. 28, 602-609 (1961)

${ }^{3}$ von Kármán, T. and Tsien, H. S., "The buckling of thin cylindrical shells under axial compression," J. Aero/Space Sci. 8, 303-312 (1941).

${ }^{4}$ Agamirov, V. L. and Volmir, A. S., "Behavior of cylindrical shells under hydrostatic dynamic loading and axial compression," Izv. Akad. Nauk SSSR, Otd. Tekhn. Nauk, Mekhan. i Mashinostr., no. 3, 78-83 (1959).

5 Timoshenko, S. and Gere, J. M., Theory of Elastic Stability (McGraw-Hill Book Co. Inc., New York, 1961).

${ }^{6}$ Arnold, R. N. and Warburton, G. B., "The flexural vibrations of thin cylinders," Proc. Inst. Eng. 167, 62-74 (1953).

${ }^{7}$ Baron, M. L. and Bleich, H., "Tables for frequencies and modes of free vibration of infinitely long thin cylindrical shells," J. Appl. Mech. 21, 178-184 (1954).

\title{
Flutter of Thin Plates under Combined Shear and Normal Edge Forces
}

\author{
J. G. EISLEY* \\ University of Michigan, Ann Arbor, Mich. \\ AND \\ G. LUESSEN† \\ The Boeing Company, Wichita, Kan.
}

\begin{abstract}
Stability boundaries are obtained for several cases of simply supported rectangular panels as follows: unbuckled plates subjected to in-plate shear, unbuckled plates under in-plane shear and normal edge forces, unbuckled plates with sweepback, and panels buckled by equal compressive stresses in both the spanwise and chordwise directions. For the unbuckled case, small deflection thin plate theory and Galerkin's method are used. Aerodynamic forces are based upon the static approximation for the most part with aerodynamic damping added in certain cases. For the buckled plate, large deflection thin plate theory and static strip theory aerodynamies are used in a static stability analysis. It has been concluded for the unbuckled plate that the shear edge loading can have a drastic effect on flutter speeds. It also was found that the use of static aerodynamic forces can lead to spurious values for the flutter boundary in certain circumstances. The addition of aerodynamic damping helps to establish the correctboundary. In the base of buckled plate, it has been concluded that the static stability boundary bears a significant relationship to the flutter boundary.
\end{abstract}

\section{Nomenclature}

$\begin{array}{ll}a, b & =\text { plate dimensions in } x \text { and } y \text { directions, respectively } \\ C_{T s} & =\text { coefficients of series expansions for unbuckled } \\ & \text { panel deflection } \\ D & =\text { plate stiffness parameter, } E h^{3} / 12\left(1-\nu^{2}\right)\end{array}$

Presented at the IAS National Summer Meeting, Los Angeles, Calif., June 19-22, 1962; revision received December 6, 1962.

* Associate Professor of Aeronautical and Astronautical Engineering.

$\dagger$ Research Specialist, Military Aircraft Systems Division.

$$
\begin{array}{ll}
E & =\text { Young's modulus for plate material } \\
h & =\text { plate thickness } \\
k, \bar{k} & =\text { frequency parameter, } \omega a^{2}\left(\rho_{s} h / D\right)^{1 / 2}, \text { and } k / \pi^{2} \\
& \quad \text { respectively } \\
M & \text { Mach number } \\
N_{x}, N_{y}, N_{x y} & =\text { normal and shear edge loads per unit length (Fig. 1) } \\
p & =\text { aerodynamic pressure } \\
q & =\text { dynamic pressure, } \frac{1}{2} \rho_{0} U^{2} \\
Q & =\lambda / \pi^{4} \\
r & =\text { aspect ratio, } b / a \\
R_{x}, R_{y}, R_{x y} & =N_{x} a^{2} / D, N_{y} a^{2} / D, \text { and } N_{x y} a^{2} / D, \text { respectively } \\
\bar{R}_{x}, \bar{R}_{y}, \bar{R}_{x y} & =R_{x} / \pi^{2}, R_{y} / \pi^{2}, \text { and } R_{x y} / \pi^{4}, \text { respectively } \\
t & =\text { time }
\end{array}
$$




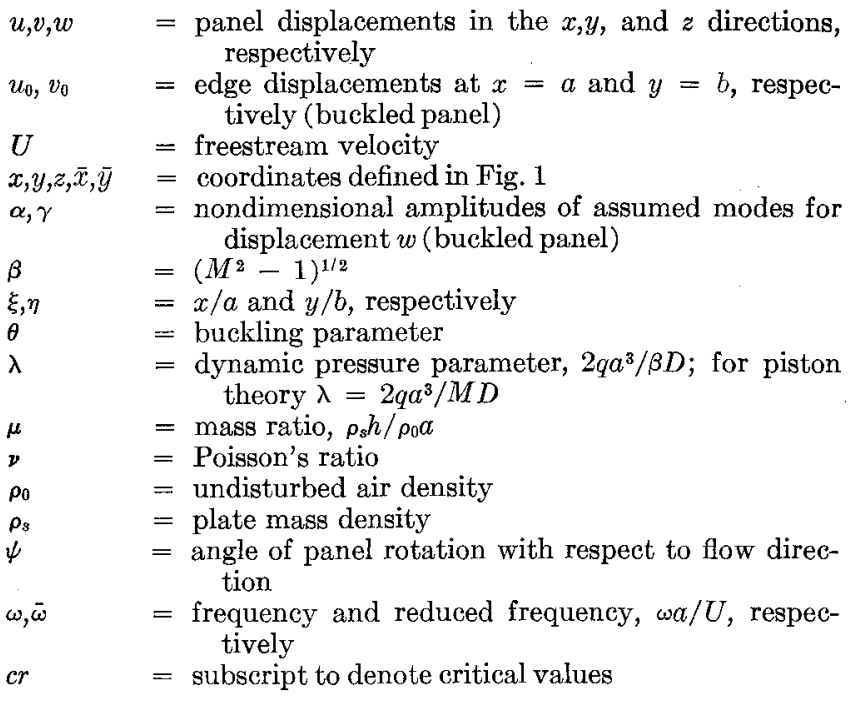

\section{Introduction}

$\mathbf{O}$ $\mathrm{NE}$ of the major obstacles to a complete understanding of supersonic panel flutter has been that the flutter boundary is sensitive to the influence of a large number of parameters. At the present time the difficulty in isolating many of these effects in an experimental study has prevented satisfactory correlation between existing experimental and theoretical results. Those problems that have been studied have been reviewed, and an extensive bibliography has been presented by Fung. ${ }^{1}$ It appears that many questions remain to be answered, even for the case of simply supported rectangular plates. No known flutter studies have included the influence of shear edge loading, for example, and information regarding the sensitivity of the stability boundary to such factors as sweepback and buckling is still incomplete.

The effects of in-plane shear are particularly significant for thin panels, since they are the shear-resistant elements of aircraft and missile structures. Also, the edges of skin panels generally will not be oriented parallel to the flow; therefore, the inclusion of sweepback, or rotation of the panel in its plane, is apropos.

Shear and sweepback have been introduced on unbuckled panels as extensions of the work of Hedgepeth ${ }^{2}$ by including additional structural and aerodynamic terms in the equations of motion. Solutions of rather large stability determinants were required to establish convergence for these results. The buckled simply supported panels have been studied to determine the effects of finite aspect ratio using a static stability analysis.

\section{Unbuckled Simply Supported Plates under Combined Shear and Normal Edge Forces with Sweepback Included}

From small deflection, thin plate theory, the nondimensional differential equation of equilibrium for a rectangular plate placed in a supersonic airstream as shown in Fig. 1 is

$$
\begin{array}{r}
\frac{\partial^{4} w}{\partial \xi^{4}}+2\left(\frac{a}{b}\right)^{2} \frac{\partial^{4} w}{\partial \xi^{2} \partial \eta^{2}}+\left(\frac{a}{b}\right)^{4} \frac{\partial^{4} w}{\partial \eta^{4}}+R_{x} \frac{\partial^{2} w}{\partial \xi^{2}}+ \\
2 R_{x y}\left(\frac{a}{b}\right) \frac{\partial^{2} w}{\partial \xi \partial \eta}+R_{y}\left(\frac{a}{b}\right)^{2} \frac{\partial^{2} w}{\partial \eta^{2}}+\frac{\rho_{s} h a^{4}}{D} \frac{\partial^{2} w}{\partial t^{2}}= \\
-\frac{a^{4}}{D} p(\xi, \eta, t)
\end{array}
$$

where $w(\xi, \eta, t)$ is the lateral deflection of the plate, $p(\xi, \eta, t)$ is the lateral aerodynamic loading, and the other quantities

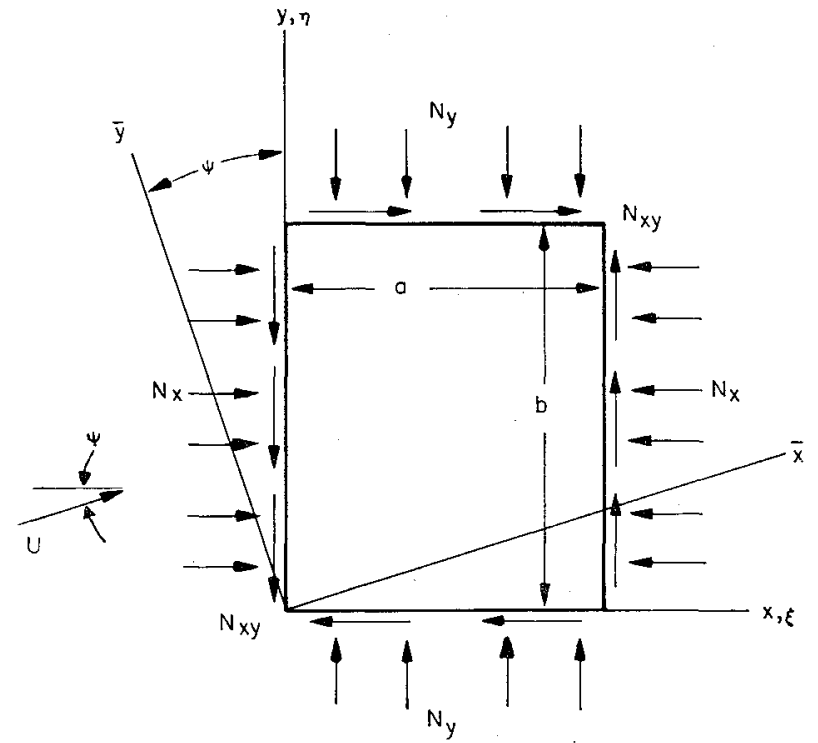

Fig. 1 Edge load and coordinate system ( $z$ axis out of page)

are defined in the Nomenclature. For simply supported plates, let

$$
w(\xi, \eta, t)=\sum_{r} \sum_{s} C_{r s} \sin r \pi \xi \sin s \pi \eta e^{i \omega t}
$$

and apply Galerkin's method. The result is

$$
\begin{gathered}
\left\{\left[m^{2}+n^{2}(a / b)^{2}\right]^{2}-\bar{R}_{x} m^{2}-\bar{R}_{y}(a / b)^{2} n^{2}-\overline{k^{2}}\right\} C_{m n}+ \\
\sum_{r} \sum_{s}\left\{8 \bar{R}_{x y}(a / b) N_{m n, r s}-\left(\lambda / \pi^{3}\right) \bar{L}_{m, r s}\right\} C_{r s}=0 \\
(m, n, r, s,=12 \ldots)
\end{gathered}
$$

where $N_{m n, r s}=4 m n r s /\left(m^{2}-r^{2}\right)\left(n^{2}-s^{2}\right)$ if $(m+r)$ and $(n+s)$ are odd, otherwise $N_{m n, r s}=0$.

The generalized airforce terms $\tilde{L}_{m n, r s}$ in Eq. (3) have been calculated using several aerodynamic theories. Static strip theory, static surface theory, and linear piston theory have been used in various situations for reasons given in the discussion of results. Static strip theory is the easiest to apply and may be obtained directly from Ref. 2 with a slight modification to account for rotation of the plate. The result is

$$
\bar{L}_{m n, r s}=\cos \psi L_{m r}+(a / b) \sin \psi L_{n s}
$$

where $L_{m r}=4 r m / \pi\left(r^{2}-m^{2}\right)$ if $(m+r)$ is odd and $n=s$, otherwise $L_{m r}=0 ; L_{n s}=4 s n / \pi\left(s^{2}-n^{2}\right)$ if $(n+s)$ is odd and $m=r$, otherwise $L_{n s}=0$. Static surface theory was used only for the nonrotated plate, and thus $\bar{L}_{m n, r s}$ may be obtained from Ref. 2 without modification. Appropriate values from linear piston theory, again for the nonrotated plate, are

$$
\bar{L}_{m n, r s}=i L_{m n}+L_{m r}
$$

where $L_{m r}$ has been defined following Eq. (4) and $L_{m n}=$ $-(\bar{\omega} / \pi)$ when $m=r$ and $n=s$, otherwise $L_{m n}=0$. It should be noted that the definition of $\lambda$ in Eq. (3) is changed slightly between the static aerodynamic theories and piston theory. For piston theory, $\beta$ is replaced by $M$, a difference that becomes less significant as $M$ increases.

The flutter boundary may be determined from Eq. (3), after including terms from the appropriate aerodynamic theory, by setting the determinant of the coefficients of $C_{m n}$ equal to zero.

For static aerodynamic theories, all roots of the characteristic equation are real for sufficiently small values of $\lambda$. If $\lambda$ then is increased gradually, a point will be reached where two roots coalesce, and any further increase in $\lambda$ makes these 


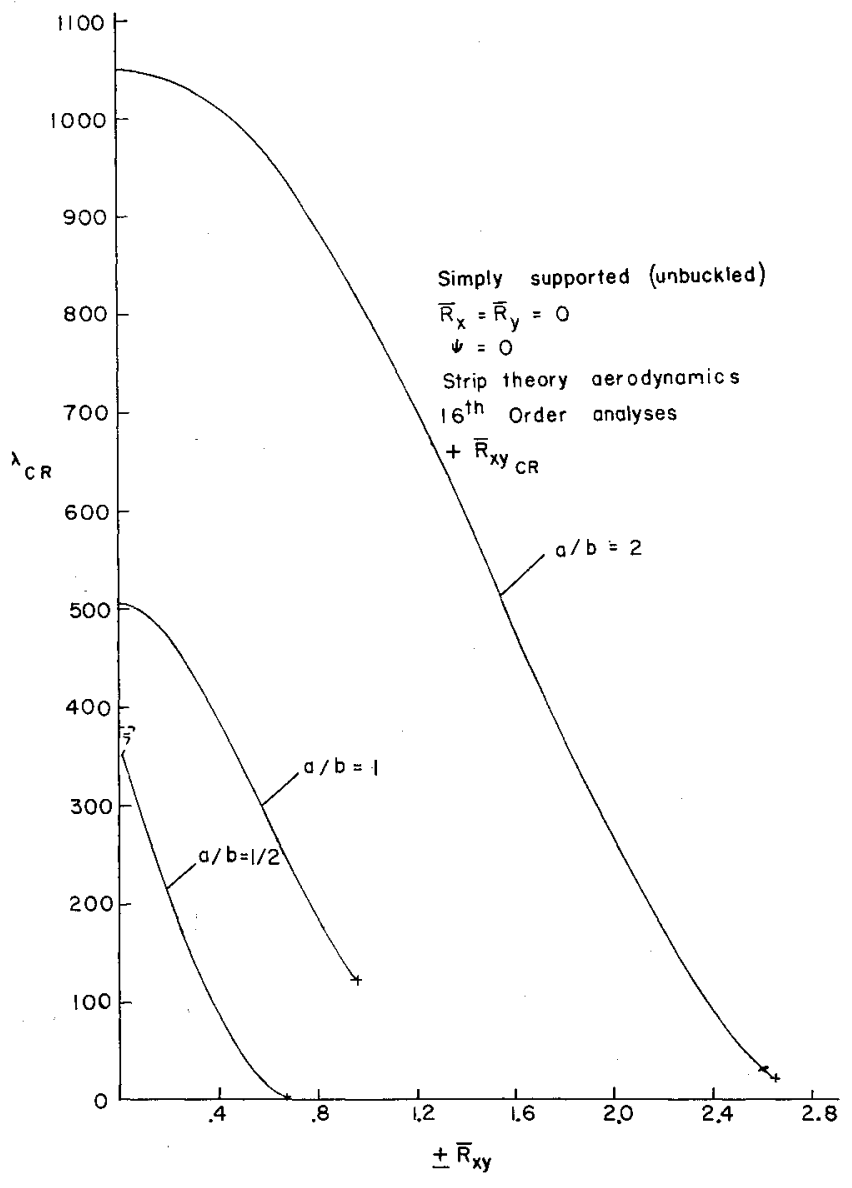

Fig. 2 Variation of critical dynamic pressure parameter with shear parameter

two roots complex. The point at which the two roots coalesce defines the critical value of $\lambda$ and hence the flutter boundary for that particular set of conditions.

For piston theory aerodynamics, the characteristic equation is complex, and the airforces are now a function of reduced frequency. Here the roots may be interpreted in a manner analogous to the classical $V-g$ flutter solutions. In order to specify this problem completely, it is necessary to fix the parameter $1 / \mu M$ in addition to those quantities that already have been specified for calculations with static strip theory.

By setting $\lambda=0$, Eq. (3) determines the natural frequencies and associated mode shapes in vacuo. Also by setting $\lambda=0$ and $\vec{k}^{2}=0$, the equations determine the critical buckling loads. The critical buckling loads are of interest, since they establish a boundary for combinations of shear and nor-

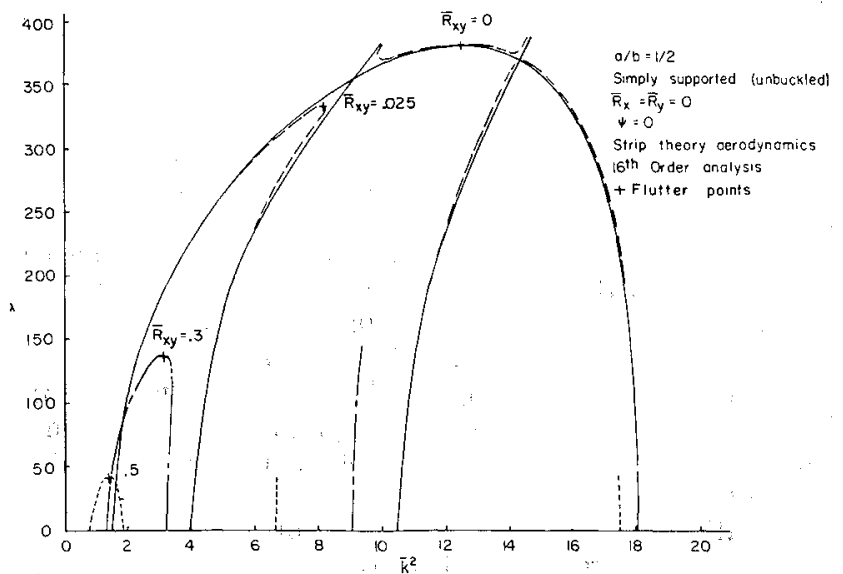

Fig. 3 Change in flutter mode for increasing values of shear parameter

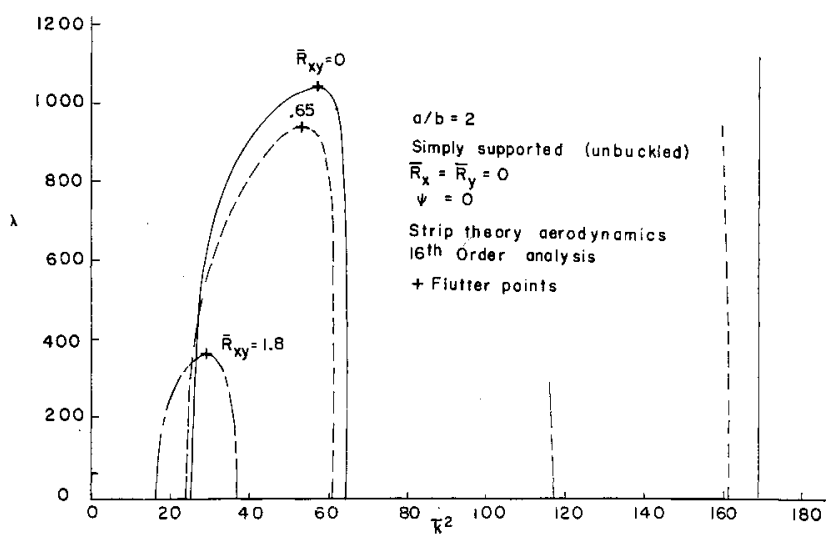

Fig. 4 Lower mode frequencies vs $\lambda$ for increasing values of shear parameter

mal edge loads beyond which small deflection theory is no longer valid. When critical edge loadings are exceeded, nonlinear equations must be used as described in the section on the stability of buckled rectangular plates.

\section{Buckled Simply Supported Plates of Finite Aspect Ratio (Static Stability)}

The static stability of a two-dimensional buckled plate in a supersonic flow has been discussed in several reports by Fung, ${ }^{3-6}$ by Eisley, ${ }^{7}$ and previous to that by Miles $^{8}$ and Isaacs. ${ }^{9}$ In the following, the static stability of a simply supported rectangular plate of finite aspect ratio in a supersonic flow is considered.

The nonlinear equations of von Karman for a thin plate with in-plane shear neglected (Fig. 1) may be written in terms of the displacements $u, v$, and $w^{10}$ as

$$
\begin{gathered}
\frac{\partial^{2} u}{\partial x^{2}}+\nu \frac{\partial^{2} v}{\partial x \partial y}+\frac{\partial w}{\partial x} \frac{\partial^{2} w}{\partial x^{2}}+\nu \frac{\partial w}{\partial y} \frac{\partial^{2} w}{\partial x \partial y}=0 \\
\frac{\partial^{2} v}{\partial y^{2}}+\nu \frac{\partial^{2} u}{\partial x \partial y}+\frac{\partial w}{\partial y} \frac{\partial^{2} w}{\partial y^{2}}+\nu \frac{\partial w}{\partial x} \frac{\partial^{2} w}{\partial x \partial y}=0 \\
D \nabla^{4} w-\frac{E h}{1-\nu^{2}}\left\{\frac{\partial u}{\partial x^{2}} \frac{\partial^{2} w}{\partial x^{2}}+\frac{1}{2}\left(\frac{\partial w}{\partial x}\right) \frac{\partial^{2} w}{\partial x^{2}}+\right. \\
\nu\left[\frac{\partial v}{\partial y} \frac{\partial^{2} w}{\partial x^{2}}+\frac{1}{2}\left(\frac{\partial w}{\partial y}\right)^{2} \frac{\partial^{2} w}{\partial x^{2}}\right]+\frac{\partial v}{\partial y} \frac{\partial^{2} w}{\partial y^{2}}+ \\
\left.\frac{1}{2}\left(\frac{\partial w}{\partial y}\right)^{2} \frac{\partial^{2} w}{\partial y^{2}}+\nu\left[\frac{\partial u}{\partial x} \frac{\partial^{2} w}{\partial y^{2}}+\frac{1}{2}\left(\frac{\partial w}{\partial x}\right)^{2} \frac{\partial^{2} w}{\partial y^{2}}\right]\right\}=-p(x, y)
\end{gathered}
$$

The boundary conditions for a simply supported rectangular plate that is buckled by displacement of the edges at $x=a$ and $y=b$ are

$$
\begin{gathered}
u(0, y)=0 \\
u(a, y)=u_{0} \\
v(x, y)=0 \quad v(x, b)=v_{0} \\
w(0, y)=w(a, y)=w(x, 0)=w(x, b)=0 \\
\frac{\partial^{2} w}{\partial x^{2}}(0, y)=\frac{\partial^{2} u}{\partial x^{2}}(a, y)=\frac{\partial^{2} w}{\partial y^{2}}(x, 0)=\frac{\partial^{2} w}{\partial y^{2}}(x, b)=0
\end{gathered}
$$

The edge displacements $u_{0}$ and $v_{0}$ will be related subsequently to the critical buckling load.

If the displacement $w$ is assumed to be

$$
w(x, y)=\alpha h \sin \frac{\pi x}{a} \sin \frac{\pi y}{b}+\gamma h \sin \frac{2 \pi x}{a} \sin \frac{\pi y}{b}
$$

the first two equations of Eqs. (6) can be solved for $u$ and $v$. If these expressions and $\mathrm{Eq}$. (8) are substituted into the third 
of Eqs. (6), and if $p(x, y)$ is chosen as the static strip theory value for supersonic flow, application of Galerkin's method gives

$$
\begin{aligned}
& \left(A_{1}+A_{2} \alpha^{2}+A_{3} \gamma^{2}\right) \alpha-\frac{8}{3}\left(\lambda / \pi^{4}\right) \gamma=0 \\
& \frac{8}{3}\left(\lambda / \pi^{4}\right) \alpha+\left(A_{4}+A_{5} \gamma^{2}+A_{3} \alpha^{2}\right) \gamma=0
\end{aligned}
$$

where

$$
\begin{aligned}
& A_{1}=(1-\theta)\left[1+\left(1 / r^{2}\right)\right]^{2} \\
& A_{2}=3\left(\frac{3-\nu^{2}}{4 r^{4}}+\frac{\nu}{r^{2}}+\frac{3-\nu^{2}}{4}\right) \\
& A_{3}=3\left(\frac{3-2 \nu^{2}}{2 r^{4}}+\frac{5 \nu}{2 r^{2}}+3-\nu^{2}\right) \\
& A_{4}=\left(\frac{1-\theta}{r^{4}}+\frac{8-5 \theta}{r^{2}}+16-4 \theta\right) \\
& A_{5}=12\left(\frac{3-\nu^{2}}{16 r^{4}}+\frac{\nu}{r^{2}}+3-\nu^{2}\right) \\
& r=b / a
\end{aligned}
$$

The symbol $\theta$ in the foregoing equations is the buckling parameter defined as

$$
\theta=u_{0} / u_{0 c r}=v_{0} / v_{0 c r}
$$

where

$$
\begin{aligned}
& u_{0 c r}=-\frac{a h^{2} \pi^{2}}{12(1+\nu)}\left(\frac{1}{a^{2}}+\frac{1}{b^{2}}\right) \\
& v_{0 c r}=-\frac{b h^{2} \pi^{2}}{12(1+\nu)}\left(\frac{1}{a^{2}}+\frac{1}{b^{2}}\right)
\end{aligned}
$$

Equation (10) is for the case of buckling under uniform edge stress in both directions such as would occur for a uniformly heated plate restrained on all edges.

For a nontrivial solution of Eqs. (9), the determinant of the coefficients of $\alpha$ and $\gamma$ is set equal to zero. After substituting

$$
\alpha^{2}=\zeta \quad \gamma^{2}=\sigma
$$

the expansion of the determinant is

$$
a_{0}+b_{0} \sigma+c_{0} \zeta+d_{0} \sigma^{2}+e_{0} \zeta \sigma+f_{0} \zeta^{2}=0
$$

where

$$
\begin{aligned}
& a_{0}=A_{1} A_{4}+\left[\frac{8}{3}\left(\lambda / \pi^{4}\right)\right]^{2} \\
& b_{0}=A_{1} A_{5}+A_{3} A_{4} \\
& c_{0}=A_{1} A_{3}+A_{2} A_{4} \\
& d_{0}=A_{3} A_{5} \\
& e_{0}=A_{2} A_{5}+A_{3}^{2} \\
& f_{0}=A_{2} A_{3}
\end{aligned}
$$

From the definitions following Eqs. (9), it may be seen that $d_{0}, e_{0}$, and $f_{0}$ are all greater than zero. Also if $\theta$ is sufficiently small but greater than one, $b_{0}$ and $c_{0}$ are greater than zero. Since for static stability both $\zeta$ and $\sigma$ must be real and positive [imaginary values of $\alpha$ and $\gamma$ would indicate that the shape assumed in Eq. (8) could not exist], it follows as a necessary condition for this special case that

$$
a_{0}<0
$$

From the definitions following Eqs. (9) and (12), the inequality expressed by Eq. (13) may be written as

$$
\theta^{2}+e_{1} \theta+e_{2}<0
$$

where

$$
\begin{aligned}
& e_{1}=-\left[20+\frac{13}{r^{2}}+\frac{2}{r^{4}}\right]\left[4+\frac{5}{r^{2}}+\frac{1}{r^{4}}\right]^{-1} \\
& e_{2}=\left[\left(16+\frac{8}{r^{2}}+\frac{1}{r^{4}}\right)+\left(1+\frac{1}{r^{2}}\right)^{-2}\left(\frac{8}{3} \frac{\lambda}{\pi^{4}}\right)^{2}\right] \times \\
& {\left[4+\frac{5}{r^{2}}+\frac{1}{r^{4}}\right]^{-1} }
\end{aligned}
$$

This inequality establishes an upper bound on the value of $\lambda$ beyond which static stability cannot exist. This maximum value of $\lambda$ will be called $\lambda_{c r}$ and is found from the relation

$$
e_{1}^{2}-4 e_{2}>0
$$

Numerical results obtained using this relation are described in Sec. IVD.

\section{Numerical Solutions}

Numerical flutter solutions have been obtained for special cases of the buckled and unbuckled simply supported panels treated in the previous sections. These results are discussed below under appropriate subheadings.

\section{A. Unbuckled Rectangular Plates with Shear Loading Only}

In order to determine the effects of shear edge forces alone,

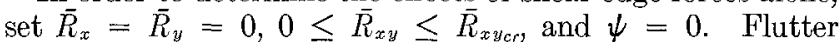
boundaries for these conditions are shown in Fig. 2 for $a / b$ of $\frac{1}{2}, 1$, and 2 . The very drastic effect on dynamic pressure parameter as shear is introduced is evident for all panel aspect ratios shown.

The unusual shape of the flutter boundary for $a / b$ of $\frac{1}{2}$ is due to the manner in which the flutter mode changes with $\bar{R}_{x y}$. This can be seen by comparing the pairs of modes whose frequencies coalesce at flutter in Figs. 3 and 4 . For $a / b$ of $\frac{1}{2}$, a variation in $\bar{R}_{x y}$ can alter the pair of modes which becomes critical, but for $a / b$ of 2 , the lowest two modes coalesce at flutter for all $\bar{R}_{x y}$. This behavior can be rationalized by an examination of the aerodynamic coupling between the vibration mode shapes both with and without shear. The area under the dashed portion of the curve for $a / b$ of $\frac{1}{2}$ (Fig. 2) is a stable region, but, for a very small amount of shear edge loading, the critical flutter speed shifts from a value in agreement with Hedgepeth ${ }^{2}$ to a significantly lower value.

\section{Number of terms required in series}

The results described previously were obtained from sixteenth-order solutions that included the first four spanwise and chordwise terms in the double sine series representing plate deflection $(m, n, r, s,=1,2,3,4)$. However, the number of terms required for satisfactory convergence has been considered, and the results of this investigation are presented in Fig. 5. The terms used in each of the solutions presented are defined in Table 1 . For an $a / b$ of 1 , the sixteenth-order solution shows satisfactory convergence when compared with the single point calculated using 24 terms. However, for an $a / b$ of $\frac{1}{2}$, the selection of terms by an orderly increase of series indices, as was done for the square plate, omits some very low frequency modes. For example, in a vacuum with zero edge loads, $\overline{k^{2}}$ for the $C_{15}$ term is 52.5 , whereas $\bar{k}^{2}$ for the $C_{4^{1}}$ term is 264 .

The normal sixteenth-order analysis excludes the $C_{15}$ term but includes the $C_{41}$ term. Therefore, an analysis was performed where terms were selected on a frequency basis. The seventeenth-order analysis shown in Table 1 was obtained for an $a / b$ of $\frac{1}{2}$ by including all terms for $\bar{k}^{2} \leq 176$ (zero edge 


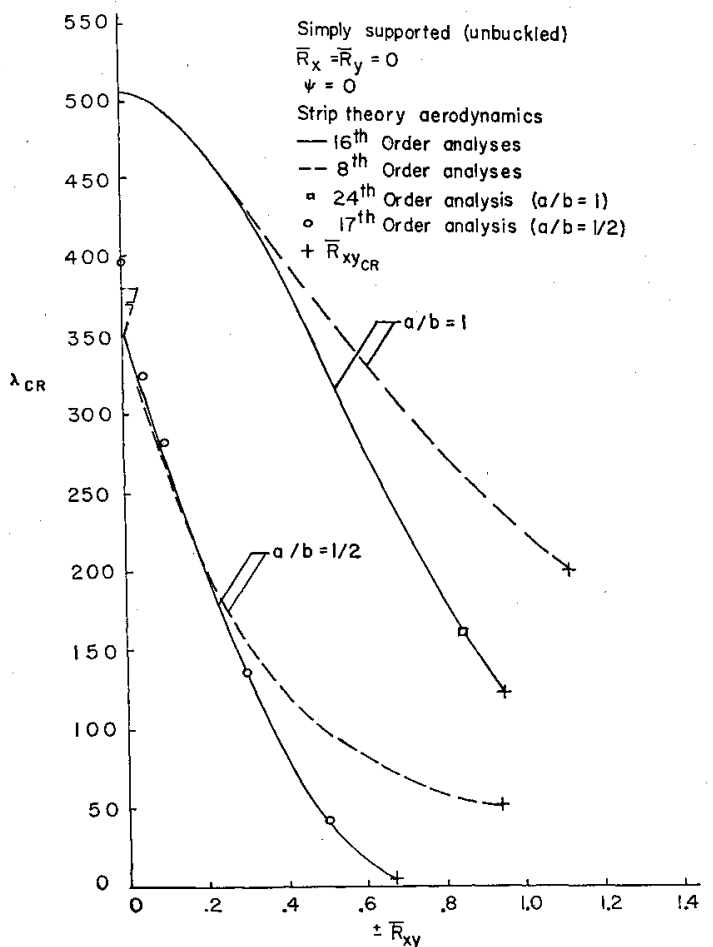

Fig. 5 Effect of the number of terms included in the series representing panel deflection

loads and airspeed). Comparison with sixteenth-order results in Fig. 5 shows virtually no difference in $\lambda_{c r}$ except at low $\bar{R}_{x y}$, where a discrepancy might have been anticipated since Hedgepeth ${ }^{2}$ shows that the $C_{41}$ term can have a significant effect on flutter speeds when shear is zero.

Another indication of having proper terms included in the analysis can be obtained from the prediction of critical buckling loads. The critical buckling loads from the sixteenth-order analyses are in good agreement with Refs. 11 and 12 , even though some higher order terms included in these references are excluded from the present analyses. The magnitudes of the coefficients omitted were small for the buckling modes shown in Refs. 11 and 12, at least for the $a / b$ reported herein, and the detrimental effect on the prediction of $\bar{R}_{x y \text { cr }}$ also was found to be small. Judging from the buckling shapes in Refs. 11 and 12, a re-examination of the terms required in the series would be desirable for a much greater range of $a / b$ than reported here. Nevertheless, all other results presented for unbuckled plates are based upon 16 terms specified by an orderly increase of series indices.

\section{Effect of surface theory aerodynamics}

It has been shown by Hedgepeth ${ }^{2}$ that strip theory is satisfactory when the plate is loaded with normal edge forces only, but it was believed appropriate to re-evaluate the effect of spanwise aerodynamic coupling with the addition of shear edge loads. Fortunately, the aerodynamic coefficients com-

Table I Terms used in series representing panel deflection

\begin{tabular}{cll}
\multicolumn{3}{c}{ for solutions of various orders } \\
\hline \hline $\begin{array}{c}\text { Order of } \\
\text { solution }\end{array}$ & $\begin{array}{c}\text { Chordwise } \\
\text { terms }(r, m)\end{array}$ & \multicolumn{1}{c}{$\begin{array}{c}\text { Spanwise } \\
\text { terms }(s, n)\end{array}$} \\
\hline 8 & $1,2,3,4$ & 1,2 \\
16 & $1,2,3,4$ & $1,2,3,4$ \\
& $\left\{\begin{array}{l}1 \\
2\end{array}\right.$ & $1,2,3,4,5,6,7$ \\
17 & 3 & $1,2,3,4,5,6$ \\
& & $1,2,4$ \\
21 & $1,2,3,4,5,6$ & $1,2,3,4$ \\
36 & $1,2,3,4,5,6$ & $1,5,6$ \\
\hline
\end{tabular}

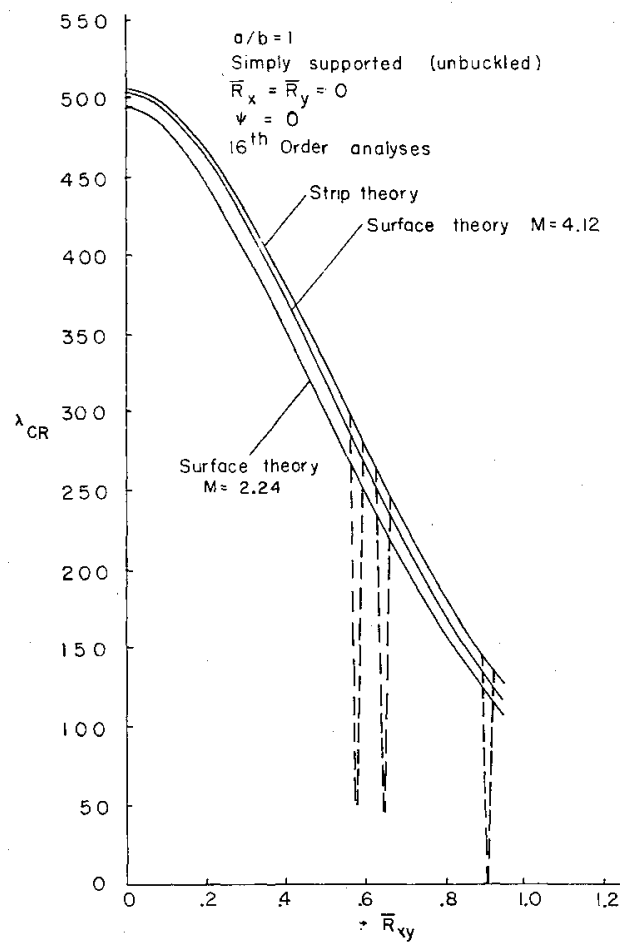

Fig. 6 Comparison of flutter boundaries for static strip and static surface theory aerodynamics

puted in Ref. 2 using the full three-dimensional static aerodynamic pressure relation could be used here. A comparison of results for strip theory and surface theory aerodynamics is shown in Fig. 6 for $M=2.24$ and 4.12. Values for strip theory aerodynamics are unconservative with respect to surface theory results by as much as $12 \%$ at $M=2.24$. However, the agreement is improved for $M=4.12$, and the flutter boundary for surface theory aerodynamics appears to be converging rapidly to that for strip theory aerodynamics as $M$ is increased.

\section{Coupling of higher modes: inclusion of aerodynamic damping}

The sharp dips in $\lambda_{c r}$ shown as dashed lines in Fig. 6 represent flutter boundaries computed in those intervals of $\bar{R}_{x y}$ for

Table 2 Vibration modes that coalesce at flutter for $\bar{R}_{x y}$ near 0.915 (also corresponding flutter modes)

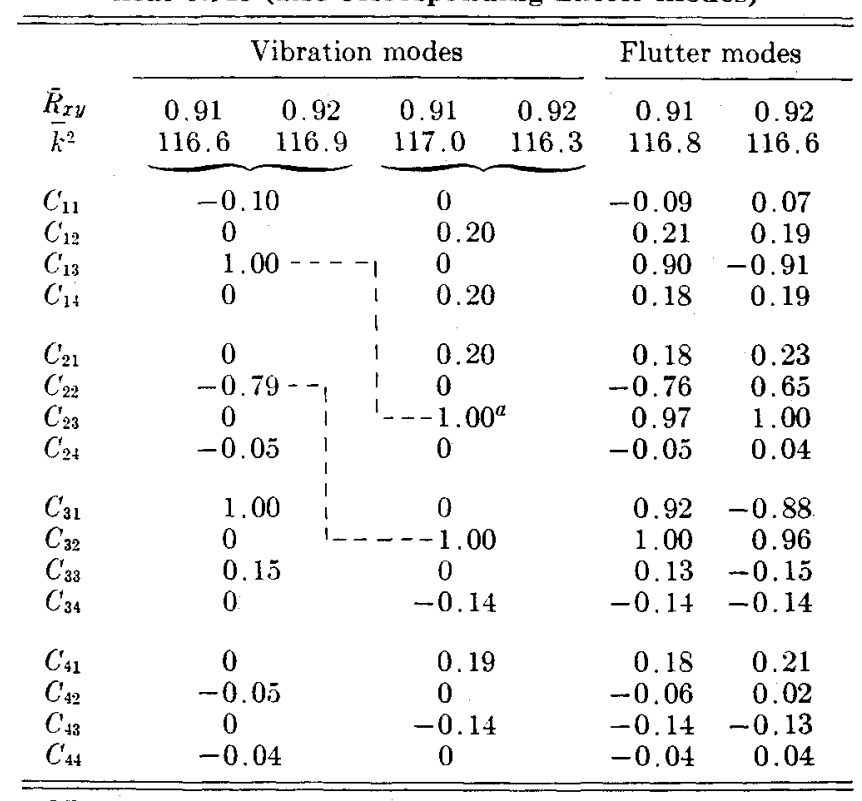

$a$ Dashed lines show aerodynamic coupling between larger terms in vibration modes. 


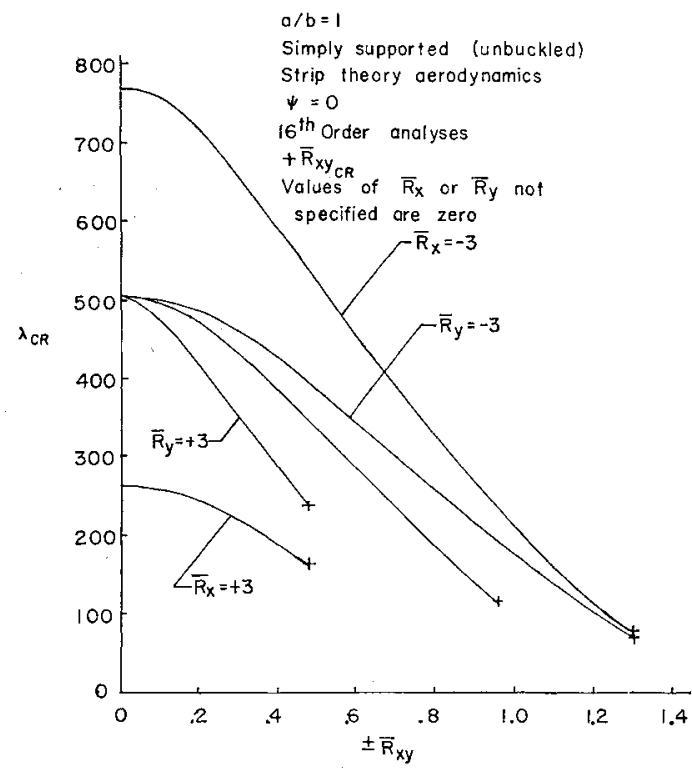

Fig. 7 Flutter boundaries with shear edge forces both with and without normal edge loads; $\overline{\boldsymbol{R}}_{x}$ or $\overline{\boldsymbol{R}}_{y}$ equal to +3 is approximately $75 \%$ critical compression load

both strip theory and surface theory aerodynamics. Examination of the changes in still air modal frequencies as the shear parameter is increased reveals that frequencies of two of the higher vibration modes cross as $\bar{R}_{x y}$ traverses the vicinity of each of the dips. For one specific value of $\bar{R}_{x y}$ in the range where each of the dips occurred, two vibration modes have identical frequencies. If these modes whose frequencies cross are aerodynamically coupled, extremely low values of $\lambda_{c r}$ might be expected (0.001 was the minimum value obtained.) That aerodynamic coupling does exist can be seen in a qualitative fashion from the modal columns shown in Table 2. The vibration modes that coalesce and the resulting flutter modes are presented for a point on each leg of the dip in $\lambda_{c r}$ for $\bar{R}_{x y}$ in the vicinity of 0.915 . It can be demonstrated easily using static strip theory that the largest terms in these vibration modes are coupled aerodynamically as indicated by the dashed lines in Table 2. Some smaller coefficients in the modal columns also contribute to the aerodynamic coupling between these modes, but the coupling shown was believed sufficient to illustrate the point. The dips in $\lambda_{c r}$ for value of $\bar{R}_{x y}$ near 0.64 and 0.59

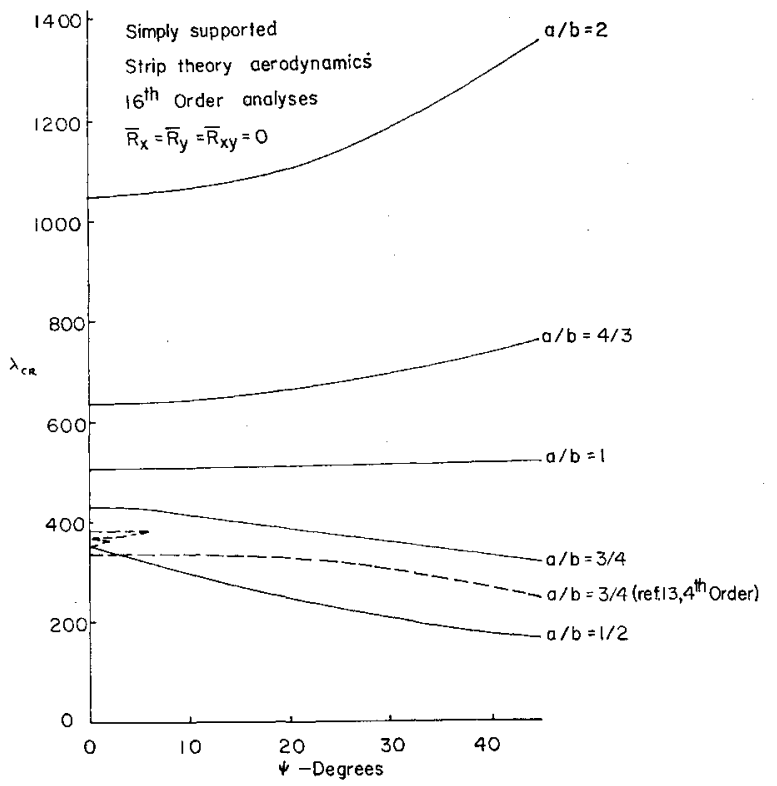

Fig. 8 Flutter boundaries for plates rotated with respect to flow

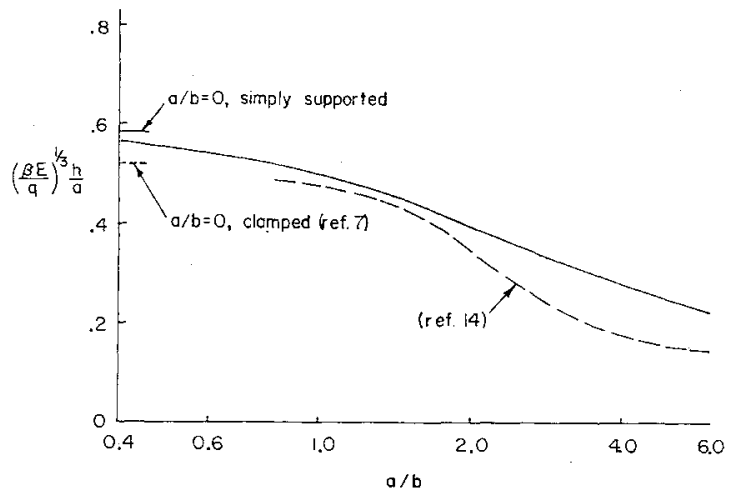

Fig. 9 Flutter boundary for buckled rectangular plates

can be rationalized using the same argument; hence the dashed boundaries in Fig. 6 appear to be legitimate flutter. boundaries for the system as represented.

Two refinements in the mathematical model were considered in an effort to eliminate the apparent drastic reductions in instability speeds. (One might imagine an even more radical boundary represented by the envelope of all dips from still higher vibration mode crossings not included in this study.) First, since the modes whose frequencies cross are of higher frequency, and hence more complex, it might be reasoned that their frequencies are not predicted accurately by the sixteenth-order analyses, and the frequency crossings in reality do not exist. For this reason, vibration analyses were performed using the 36 -term representation for plate deflection shown in Table 1. Results from the iterative solutions showed that, although the frequencies of the higher modes were lowered somewhat, the frequency crossings still were present.

The second possibility considered was to add aerodynamic damping to the system by inserting the expression for piston theory aerodynamics, Eq. (5), into Eq. (3). Solutions using piston theory did produce the desired smooth flutter boundaries, and although the curve for strip theory on Fig. 6 was not duplicated the results appeared to be converging to this boundary with increasing values of the parameter $\mu M$. Therefore, the possibility of sharp dips occurring was ignored subsequently whenever frequencies of higher modes were nearly equal.

The flutter modes included in Table 2 are shown as a matter of interest. Since these flutter modes are almost a direct sum of the two vibration modes tabulated, they clearly show the phasing differences between vibration modes at flutter when $\vec{R}_{x y}$ equals 0.91 or 0.92 . Presumably, the phase shift is caused by the crossing of frequencies of these two modes as $\bar{R}_{x y}$ is increased from 0.91 to 0.92 (zero airspeed). Hence, for frequency coalescence of these vibration modes, the sign of the aerodynamic stiffnesses (coupling terms) must change when the lower and higher frequency modes are interchanged.

\section{B. Unbuckled Rectangular Plates under Combined Shear and Normal Edge Loads}

To evaluate the effect of shear edge loads in the presence of normal edge forces in both the chordwise and spanwise directions, a series of computations was made as follows $\left(\psi=0 ; 0 \leq \bar{R}_{x y} \leq \bar{R}_{x y c r}\right)$ :
1) $\bar{R}_{x}=0$
2) $\bar{R}_{x}=0$
3) $\bar{R}_{x}=0.75 \bar{R}_{x c r}$ (approx.)
$\bar{R}_{y}=0.75 \bar{R}_{y_{\bar{c} r}}$ (approx.)
4) $\bar{R}_{x}=-0.75 \bar{R}_{x_{c r}}$ (approx.)
$\bar{R}_{y}=\overline{0}$
$\bar{R}_{y}=0$$$
\bar{R}_{y}=0
$$

$\bar{R}_{x_{c r}}$ is the critical compressive load for $\bar{R}_{y}=0$ and $\bar{R}_{x y}=0$; $\bar{R}_{y_{c r}}$ is defined in an analogous fashion, and $\bar{R}_{x y_{c r}}$ is the critical 
shear load for the particular normal edge loading specified. The results from these calculations for $a / b$ of 1 are compared in Fig. 7 with those previously described for no normal edge loading. The detrimental effect of shear edge loads when combined with either spanwise or chordwise edge loading is evident for this aspect ratio. It has been concluded in Ref. 2 that the flutter boundary is unaffected by spanwise normal edge loads, but this is shown to be no longer true when shear loading is added. Generally speaking, tension edge loads are beneficial, whereas compression normal edge loads are detrimental, with the loads applied in the chordwise direction being the most influential. As the critical values of shear are approached, it makes less difference whether the normal edge loading is in the chordwise or spanwise direction.

\section{Unbuckled Rectangular Plates with Sweepback}

The flutter of unbuckled rectangular plates rotated with respect to flow direction has been investigated for zero shear and normal edge forces. For this case $\bar{R}_{x}=\bar{R}_{y}=\bar{R}_{x y}=0$ and $\psi \neq 0$. These results are shown in Fig. 8 for flow angles from 0 to $\pi / 4$ and several values of $a / b$. The range of $\psi$ is not extended beyond $\pi / 4$, since $\lambda$ then would be based upon a panel dimension that is oriented more in the spanwise direction than in the chordwise sense. Generally speaking, sweepback is seen to be detrimental for low $a / b$ and beneficial for higher values of $a / b$.

The dashed continuation of the flutter boundary for $a / b$ of $\frac{1}{2}$ is somewhat analogous to that already described for this same $a / b$ with shear edge loading. The variation of modal frequencies with dynamic pressure parameter shown in Fig. 3 for $\bar{R}_{x y}=0$ applies here for $\psi=0$. If the panel is rotated a small amount, the spanwise component of flow couples the two lowest frequency modes $\left(C_{11}\right.$ and $\left.C_{12}\right)$ that are uncoupled for zero sweepback, and a plot of $\overline{k^{2}}$ vs $\lambda$ would be similar to that shown for small $\bar{R}_{x y}$. It is interesting to note that a small amount of either shear or sweepback produces the same drop in $\lambda_{c r}$ from the zero shear, zero sweepback case. This appears to be another example of the delicate nature of panel flutter, and unless the complete absence of shear and sweepback is insured it would seem more rational to use the lower curve to define the flutter boundary.

During the time that this investigation was being performed, Ref. 13 was published which in essence treats this same special case of the more general problem described in the flutter theory for unbuckled panels. Reference 13 contains results for a rather complete variation of panel aspect ratio; however, only four terms were used in the series representing plate deflection. Although proper trends are computed with the lower order analyses, Fig. 8 shows by comparison with the results for sixteenth-order analyses that significant changes in flutter boundaries are obtained with the inclusion of higher terms. The sixteenth-order analyses used herein are not regarded as having a minimum number of terms required for convergence, but the results are believed to represent converged boundaries for practical purposes.

\section{Buckled Rectangular Plates}

The theory developed for buckled plates treats the case where the plate is buckled by uniform compressive stresses of equal magnitudes in the spanwise and chordwise directions. This type of loading is of practical significance, since it represents the stresses that would occur in a uniformly heated plate restrained on all edges. For $r \rightarrow \infty(a / b \rightarrow 0)$, the inequality of Eq. (15) establishes that

$$
\lambda_{c r}=109.6
$$

or

$$
Q_{c r}=\lambda_{c r} / \pi^{4}=1.125
$$

No statically stable configuration exists for larger values of $\lambda$. This agrees with the value obtained by Fung ${ }^{3-6}$ for a two-mode solution of the infinite aspect ratio case.

Values of $\lambda_{v r}$ for various values of $a / b$ have been determined, and the results are plotted in Fig. 9 together with the envelope of experimental results given in Ref. 14. In addition, the critical value obtained in Ref. 7 by a two-mode solution for infinite aspect ratio clamped plates is shown. The ordinate for these curves is one that frequently is used to present experimental data, and for this parameter the region below the curves is unstable.

It should be noted that the effects of the use of strip theory aerodynamics become more pronounced as $a_{0} / b$ gets larger. Also, the problem of convergence has not been settled; however, in Ref. 6 it is shown that a six-mode solution of the infinite aspect ratio case results in $Q_{c r}=1.172$, which indicates that the first two modes are sufficient as a first approximation.

From these results, it appears that the static stability boundary has practical significance in relation to the flutter boundary. A full dynamic analysis is in preparation.

\section{References}

${ }^{1}$ Fung, Y. C., "A summary of the theories and experiments on panel flutter," Air Force Office Sci. Research TN 60-224 (May $1960)$.

2 Hedgepeth, J. M., "Flutter of rectangular simply supported panels at high supersonic speeds," J. Aeronaut. Sci. 24, 563573 (1957).

3 Fung, Y. C., "On two-dimensional panel flutter," J. Aeronaut. Sci. 25, 145-160(1958).

${ }^{4}$ Fung, Y. C., "Flutter of curved plates with edge compression in a supersonic flow,' Proceedings of the Midwest Conference on Fluid and Solid Mechanics, Ann Arbor, Mich., AFOSR TN 57187 (April 1957).

5 Fung, Y. C., "The flutter of a buckled plate in a supersonic flow," Rept. OSR TN 55-237, Guggenheim Aeronaut. Lab., Calif. Inst. Tech. (July 1955).

${ }^{6}$ Fung, Y. C., "The static stability of a two-dimensional curved panel in a supersonic flow with an application to panel flutter," J. Aeronaut. Sci. 21, 556-565 (1954).

${ }^{7}$ Eisley, J. G., "Flutter of a two-dimensional buckled plate in a supersonic flow," Rept. OSR TN 56-296, Guggenheim Aeronaut. Lab., Calif. Inst. Tech. (July 1956).

${ }^{8}$ Miles, J. W., "Dynamic chordwise stability at supersonic speeds," Rept. AL-1140, North American Aviation Inc. (October 1950).

${ }^{9}$ Isaacs, R. P., "Transtability flutter of supersonic aircraft panels," Rand Rept. P-101 (July 1949).

${ }^{10} \mathrm{Chu}, \mathrm{H}$. N. and Herrman, G., "Infuence of large amplitudes on free flexural vibrations of rectangular elastic plates," J. Appl. Mech. 23, 532-540 (December 1956).

11 Stein, M. and Neff, J., "Buckling stresses of simply supported rectangular flat plates in shear," NACA TN 1222 (March 1947).

${ }^{12}$ Batdorf, S. B. and Stein, M., "Critical combinations of shear and direct stress for simply supported rectangular flat plates," NACA TN 1223 (March 1947).

${ }^{13}$ Kordes, E. E. and Noll, R. B., "Theoretical flutter analysis of flat rectangular panels in uniform coplanar flow with arbitrary direction," NASA TN-1156 (January 1962).

${ }^{14}$ Kordes, E. E., Tuovila, W. J., and Guy, L. D., "Flutter research on skin panels," NASA TN D-451 (September 1960). 